\title{
Surgical Outcomes for Obstructive Total Anomalous Pulmonary Venous Return of the Non-Infracardiac Type Compared with the Infracardiac Type
}

\author{
Jae Yoon Na, ${ }^{1}$ Jinyoung Song, ${ }^{1}$ June Huh, ${ }^{1}$ I-Seok Kang, ${ }^{1}$ Ji-Hyuk Yang, ${ }^{2}$ Tae-Gook Jun ${ }^{2}$ \\ ${ }^{1}$ Department of Pediatrics, Samsung Medical Center, Sungkyunkwan University School of Medicine, Seoul, Korea; \\ ${ }^{2}$ Department of Thoracic and Cardiovascular Surgery, Samsung Medical Center, Sungkyunkwan University School of Medicine, Seoul, Korea
}

\section{ABSTRACT}

Background: Infracardiac obstructive total anomalous pulmonary venous return (TAPVR) has a poor outcome following surgical correction. We compared the surgical outcomes of obstructive TAPVR between non-infracardiac and infracardiac types.

Methods: Among 51 patients who underwent surgical repair for obstructive TAPVR, 23 with infracardiac type and 28 with non-infracardiac type were included in this investigation. The study compared the immediate postoperative courses in the intensive care unit and long-term mortality and pulmonary vein stenosis. The risk factors for long-term survival in obstructive TAPVR also were investigated.

Results: The postoperative follow-up period was 79.8 \pm 81.5 months. Immediate major operative complications were observed in 22 patients (43.1\%); 10 patients $(19.6 \%)$ died, and eight patients (15.7\%) experienced pulmonary vein stenosis during the follow-up period. The KaplanMeier curve showed better cumulative survival in patients with infracardiac TAPVR $(P=0.308)$. The significant factors for survival after surgical repair of obstructive TAPVR did not include anatomical type but instead were postoperative course of ventilator care and lengths of intensive care unit and hospital stays.

Conclusion: Patients with non-infracardiac TAPVR with obstruction had a longer postoperative course and experienced more complications. Their survival rate was poorer, and postoperative pulmonary vein stenosis was more frequent in those patients compared with infracardiac TAPVR patients. However, a large-scale study is mandatory to gather more data and confirm our findings.

\section{INTRODUCTION}

Total anomalous pulmonary venous return (TAPVR) is one of the most fatal congenital heart diseases. If not repaired

Received fuly 23, 2020; accepted September 11, 2020.

Correspondence: Finyoung Song, MD, PhD, Department of Pediatrics, Samsung Medical Center, Heart Vascular Stroke Institute, Grown-up Congenital Heart Clinic, Sungkyunkwan University School of Medicine, 81 Irwon-ro, Gangnamgu, Seoul 06351, Korea, Telephone +82-2-3410-3539, Fax +82-2-3410-0043 (e-mail: amyjys@naver.com) within the first month of life, obstructive TAPVR typically is symptomatic and often leads to death [Michielon 2002]. There has been remarkable improvement in surgical results and development of preoperative diagnostic methods, operative techniques, and postoperative management strategies [Anil Kumar 2003]. There are four types of TAPVR classified based on the involved connecting vein: supracardiac, cardiac, infracardiac, and mixed [Shaw 2017]. The infracardiac type is most frequently associated with obstruction that accounts for poor patient outcomes following surgical repair [Hawkins 1995; Kirshbom 2002; Hancock Friesen 2005; Karamlou 2007; Seale 2010; Yong 2011]. However, the treatment results in obstructive TAPVR patients with types other than infracardiac are not well known. Therefore, the aim of this study was to evaluate the immediate and midterm results of total repair for obstructive TAPVR and to compare these results in non-infracardiac and infracardiac TAPVR patients.

\section{METHODS}

\section{Patients}

We performed a retrospective study of all patients who underwent surgery for obstructive TAPVR at Samsung Medical Center between January 1995 and December 2018. All cases that were comorbid with other major congenital heart diseases (single ventricle, atrial isomerism, hypoplastic left heart syndrome, double outlet right ventricle, complete atrioventricular septal defect, and pulmonary atresia with ventricular septal defect, etc.) were excluded.

Study design and data collection: Patients were classified into four groups, according to TAPVR type as defined using the Craig and Darling Classification system [Craig 1957]: supracardiac, intracardiac, infracardiac, and mixed. Further division was carried out into infracardiac and non-infracardiac types, depending on obstruction site. Preoperative demographic data and information regarding the postoperative hospital course, such as duration of ventilator care, duration of intensive care unit (ICU) stay, and total duration of hospitalization, were compiled by review of medical records. Major complications were defined as various postoperative events that required surgical or medical intervention, such as chylothorax, diaphragmatic palsy, arrhythmia, and pleural effusion, which were treated with surgery or medication. Postoperative pulmonary vein stenosis on echocardiography, 
early hospital mortality defined as death during initial hospitalization or within the first 30 postoperative days, and longterm mortality also were assessed.

\section{Statistical analysis}

Data are given as frequency, median with minimum and maximum value, or mean \pm standard deviation, as appropriate. Patient survival time was evaluated using Kaplan-Meier curves based on whether the TAPVR was infracardiac or non-infracardiac. Relative risk for survival was evaluated by Cox's proportional hazards model. For comparison between two groups, $\chi 2$ and Student's t-test were used, when appropriate. All data were analyzed using the Statistical Package for the Social Sciences (SPSS), version 25.0 (IBM Corp., Armonk, NY, USA). A $P$ value $>0.05$ was accepted as statistically significant. This study was approved by the Institutional Review Board of Samsung Medical Center, and the need for informed consent was waived, due to its retrospective nature.

\section{RESULTS}

All data for patients with obstructive TAPVR are presented in Table 1. (Table 1) Overall, 149 patients were diagnosed with TAPVR and underwent surgery; only 51 of these patients with isolated TAPVR and pulmonary venous obstruction as diagnosed using two-dimensional echocardiography were enrolled in this study. Of these 51 patients, 19 were female, and 32 were male. Operation age and body weight were $21.3 \pm 30.9$ days and $3.4 \pm 0.7 \mathrm{~kg}$, respectively. The surgical procedure was performed successfully for supracardiac (22 patients), cardiac (four patients), mixed (six patients), and infracardiac (19 patients) types. In six mixed TAPVR cases, three of four pulmonary veins drained into the portal vein in four patients, so that those four cases belonged to infracardiac type. Therefore, 51 patients with obstructive TAPVR were divided into infracardiac TAPVR (23 patients) and noninfracardiac TAPVR (28 patients) types.

The length of the postoperative follow-up period was $79.8 \pm 81.5$ months. The mean postoperative periods, where ventilator care and intensive care were required, were 13.9 and 20.0 days, respectively. Immediate major operative complications, including diaphragmatic palsy, chylothorax, pleural effusion, arrhythmia, and pump weaning failure, were observed in 22 patients $(43.1 \%)$. (Table 2) Seven patients died early in the postoperative course. Three of these seven patients were of the infracardiac type, and the remaining four had non-infracardiac type TAPVR. One patient died due to postoperative brain damage, while the other six died from acute respiratory failure due to pulmonary hypertensive crisis. After initial hospitalization or after the first 30 postoperative days, there were no deaths from infracardiac TAPVR, but three patients with non-infracardiac TAPVR died. Overall, 10 patients (19.6\%) died, and eight additional patients $(15.7 \%)$ suffered from pulmonary vein stenosis, during the follow-up period. The Kaplan-Meier curve showed a better cumulative survival time in patients with infracardiac TAPVR than for those with non-infracardiac TAPVR, but this difference was not statistically significant $(P=0.308)$. (Figure 1)

According to Cox's proportional hazards model, the significant factors for survival after repair of obstructive TAPVR did not include anatomical type of TAPVR, but instead postoperative course of ventilator care, ICU stay, and total hospital stay. (Table 3) Finally, our study revealed significantly longer hospitalization in patients with non-infracardiac TAPVR compared with infracardiac TAPVR.

Table 1. Data for all patients after the repair of obstructive total anomalous pulmonary venous return (TAPVR)

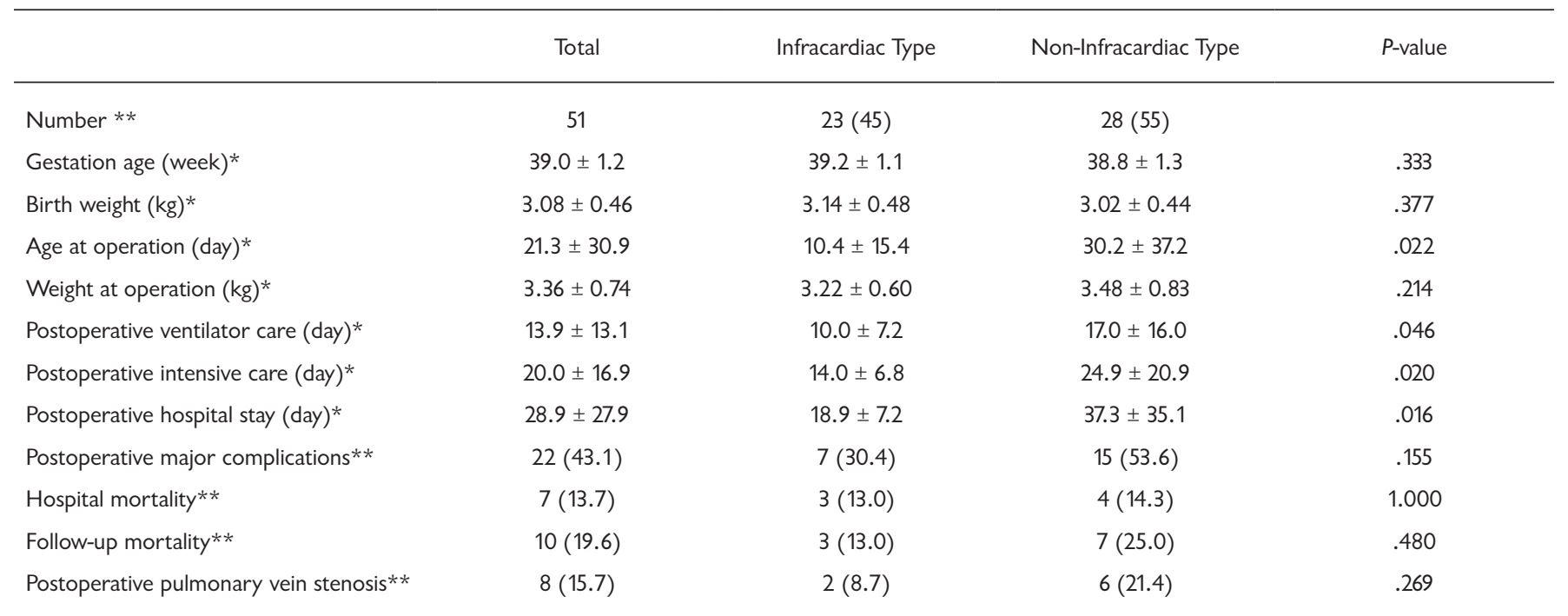

* Mean $\pm S D, * * N(\%)$ 
Table 2. Postoperative major complications

\begin{tabular}{lccc}
\hline & & & Non-Infracardiac \\
& Total & Infracardiac Type & Type \\
\hline Diaphragmatic palsy (n) & 5 & 1 & 4 \\
Chylothorax (n) & 4 & 1 & 3 \\
Pleural effusion (n) & 7 & 3 & 4 \\
Arrhythmia (n) & 5 & 2 & 3 \\
ECMO insertion (n) & 1 & 0 & 1 \\
\hline
\end{tabular}

$\mathrm{n}$ : number of patients

\section{DISCUSSION}

Our study suggested that patients with non-infracardiac TAPVR with obstruction had a longer postoperative course and more complications than the other group. In addition, the survival rate was poorer, and the postoperative pulmonary vein stenosis rate was more frequent in these patients compared with infracardiac TAPVR patients with obstructive pulmonary vein stenosis, although this difference was not statistically significant.

The mortality rate, following repair of isolated TAPVR, has decreased dramatically [Karamlou 2007; Modi 2008; Lemaire 2017]. However, preoperative pulmonary vein obstruction and infracardiac TAPVR have been suggested as risk factors for early mortality in various studies [Karamlou 2007; Husain 2012; Lemaire 2017; Shi 2017]. In obstructive TAPVR, Jinghao et al. reported an operative mortality of $2.9 \%$ [Jinghao 2014], whereas Huiwen et al. found an overall mortality rate of $26.7 \%$ in the preoperative obstructive type [Shi 2017]. Based on our study's results, length of hospital stay and longterm mortality in obstructive TAPVR were $13.7 \%$ and $19.6 \%$, respectively, and significant risk factors for survival according to a Cox's proportional hazards model were duration of postoperative ventilator care, length of ICU stay, and hospitalization duration but not anatomical type of TAPVR. Therefore, the overall outcome of repair for obstructive TAPVR is likely associated with the immediate postoperative course.

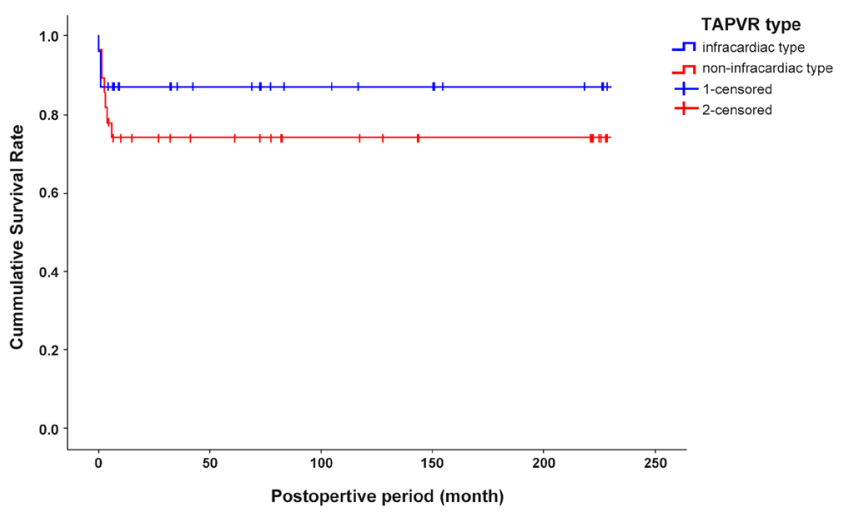

Figure 1. The Kaplan-Meier cumulative survival curve revealed a better survival rate postoperatively in patients with infracardiac obstructive TAPVR than in patients with non-infracardiac obstructive TAPVR. TAPVR; total anomalous pulmonary venous return.

Previous studies have reported that infracardiac TAPVR with obstruction carries a higher mortality than noninfracardiac TAPVR with obstruction [Jinghao 2014; Shi 2017]. However, we observed that the immediate postoperative course was significantly worse in patients with noninfracardiac TAPVR based upon longer length of ICU stay, ventilator care requirements, and overall length of hospitalization. Although the difference was not statistically significant, major postoperative complications and pulmonary vein stenosis were more frequent in patients with noninfracardiac TAPVR. In addition, higher total mortality and lower cumulative survival rates were observed in patients with non-infracardiac type. We suggest that this trend might be associated with time pulmonary venous obstruction occurred. Due to patent ductus venosus during the fetal period, pulmonary venous obstruction in infracardiac type patients might only present after birth, and pulmonary congestion then progresses rapidly after closure of the ductus venosus at birth. However, pulmonary venous obstruction in non-infracardiac type patients is not related to patent ductus venosus and can therefore exist during the fetal period. The successful stenting of ductus venosus soon after birth in two

Table 3. Relative risk (Cox's proportional hazards model) for survival after repair of obstructive TAPVR

\begin{tabular}{|c|c|c|c|c|c|c|c|c|}
\hline & B & SE & Wald & df & sig & $\operatorname{Exp}(B)$ & lower & upper \\
\hline Age at operation & -0.186 & 0.202 & 0.847 & 1 & 0.357 & 0.83 & 0.558 & 1.234 \\
\hline Weight at operation & -1.107 & 1.372 & 0.651 & 1 & 0.420 & 0.33 & 0.022 & 4.867 \\
\hline Postoperative hospital stay & 0.126 & 0.043 & 8.675 & 1 & 0.003 & 1.13 & 1.043 & 1.233 \\
\hline TAPVR type & -0.0687 & 0.690 & 0.990 & 1 & 0.320 & 0.503 & 0.130 & 1.946 \\
\hline
\end{tabular}


neonates, who demonstrated a good outcome, supports our hypothesis [Higaki 2010].

This study had the usual limitations of a retrospective, non-randomized investigation. Because this study was a single institutional study, the treatment protocol and surgical techniques were likely uniform, which made it is easy to analyze the data. However, due to the small number of patients, statistical significance could not be determined. And there are many confounding factors that might make different surgical outcomes, such as limitations in diagnosis, time of surgery, different surgical techniques, and patient's characteristics. Some patients were lost to follow up and other reasons for bias can exist in this study. Therefore, a large-scale, wellcontrolled study will be required to support the findings of inferior outcomes in repair of non-infracardiac TAPVR.

\section{CONCLUSION}

Although this is a limited study with small number of patient, the outcomes of surgical repair for obstructive TAPVR of the non-infracardiac type were not better than those of infracardiac type. A longer postoperative course and more complications were observed in patients with non-infracardiac TAPVR with obstruction. Their survival rate was poorer, and postoperative pulmonary vein stenosis was more frequent in those patients compared with infracardiac TAPVR. However, a large-scale and well designed study is mandatory for support our findings.

\section{REFERENCES}

Anil Kumar D, Kumar RN, Narasinga Rao P, et al. 2003. Repair of total anomalous pulmonary venous connection in early infancy. Asian Cardiovasc Thorac Ann 11:18-22.

Craig JM, Darling RC, Rothney WB. 1957. Total pulmonary venous drainage into the right side of the heart; report of 17 autopsied cases not associated with other major cardiovascular anomalies. Lab Invest 6:44-64.

Hancock Friesen CL, Zurakowski D, Thiagarajan RR, et al. 2005. Total anomalous pulmonary venous connection: an analysis of current management strategies in a single institution. Ann Thorac Surg 79:596-606; discussion 596-606.
Hawkins JA, Minich LL, Tani LY, Ruttenberg HD, Sturtevant JE, McGough EC. 1995. Absorbable polydioxanone suture and results in total anomalous pulmonary venous connection. Ann Thorac Surg 60:55-9.

Higaki T, Yamamoto E, Nakano T, et al. 2010. Successful stenting of the ductus venosus in 2 neonates with asplenia syndrome complicated by infracardiac type total anomalous pulmonary venous connection. J Cardiol Cases 1:e129-e32.

Husain SA, Maldonado E, Rasch D, et al. 2012. Total anomalous pulmonary venous connection: factors associated with mortality and recurrent pulmonary venous obstruction. Ann Thorac Surg 94:825-31; discussion 31-2.

Jinghao Z, Botao G, Zhiwei X, Jinfeng L. 2014. The research on operation of obstructed total anomalous pulmonary venous connection in neonates. Scientific World Journal 2014:576569.

Karamlou T, Gurofsky R, Al Sukhni E, et al. 2007. Factors associated with mortality and reoperation in 377 children with total anomalous pulmonary venous connection. Circulation 115:1591-8.

Kirshbom PM, Myung RJ, Gaynor JW, et al. 2002. Preoperative pulmonary venous obstruction affects long-term outcome for survivors of total anomalous pulmonary venous connection repair. Ann Thorac Surg 74:1616-20.

Lemaire A, DiFilippo S, Parienti JJ, et al. 2017. Total Anomalous Pulmonary Venous Connection: A 40 years' Experience Analysis. Thorac Cardiovasc Surg 65:9-17.

Michielon G, Di Donato RM, Pasquini L, et al. 2002. Total anomalous pulmonary venous connection: long-term appraisal with evolving technical solutions. Eur J Cardiothorac Surg 22:184-91.

Modi A, Vohra HA, Brown I, Langley SM. 2008. Successful surgical correction of total anomalous pulmonary venous drainage in the sixth decade. Ann Thorac Surg 86:1684-6.

Seale AN, Uemura H, Webber SA, et al. 2010. Total anomalous pulmonary venous connection: morphology and outcome from an international population-based study. Circulation 122:2718-26.

Shaw FR, Chen JM. 2017. Surgical Considerations in Total Anomalous Pulmonary Venous Connection. Semin Cardiothorac Vasc Anesth 21:132-37.

Shi G, Zhu Z, Chen J, et al. 2017. Total Anomalous Pulmonary Venous Connection: The Current Management Strategies in a Pediatric Cohort of 768 Patients. Circulation 135:48-58.

Yong MS, d'Udekem Y, Robertson T, et al. 2011. Outcomes of surgery for simple total anomalous pulmonary venous drainage in neonates. Ann Thorac Surg 91:1921-7. 